\title{
Multifunctional targeting micelle nanocarriers with both imaging and therapeutic potential for bladder cancer
}

This article was published in the following Dove Press journal:

International Journal of Nanomedicine

2 June 2012

Number of times this article has been viewed

\author{
Tzu-yin Lin' \\ Hongyong Zhang' \\ Juntao Luo ${ }^{2,5}$ \\ Yuanpei $\mathrm{Li}^{2}$ \\ Tingjuan $\mathrm{Gao}^{3}$ \\ Primo N Lara Jr ${ }^{1,4,6}$ \\ Ralph de Vere White ${ }^{4}$ \\ Kit S Lam ${ }^{1,2}$ \\ Chong-Xian Pan ${ }^{1,3,6}$ \\ 'Division of Hematology and \\ Oncology, Department of Internal \\ Medicine, ${ }^{2}$ Department of \\ Biochemistry and Molecular Medicine, \\ ${ }^{3}$ NSF Center for Biophotonics \\ Science and Technology, School \\ of Medicine, ${ }^{4}$ Department of \\ Urology, University of California- \\ Davis, Sacramento, CA, ${ }^{5}$ Department \\ of Pharmacology, SUNY Upstate \\ Medical University, Syracuse, NY, \\ ${ }^{6}$ VA Northern California Health Care \\ System, Mather, CA, USA
}

Correspondence: Chong-Xian Pan Division of Hematology/Oncology, Department of Internal Medicine, University of California at Davis, 4501 X Street, Room 3016, Sacramento, CA 95817, USA

$\mathrm{Tel}+\mid 916734377$ I

Fax + I 9167347946

Email cxpan@ucdavis.edu
Background: We previously developed a bladder cancer-specific ligand (PLZ4) that can specifically bind to both human and dog bladder cancer cells in vitro and in vivo. We have also developed a micelle nanocarrier drug-delivery system. Here, we assessed whether the targeting micelles decorated with PLZ4 on the surface could specifically target dog bladder cancer cells.

Materials and methods: Micelle-building monomers (ie, telodendrimers) were synthesized through conjugation of polyethylene glycol with a cholic acid cluster at one end and PLZ4 at the other, which then self-assembled in an aqueous solution to form micelles. Dog bladder cancer cell lines were used for in vitro and in vivo drug delivery studies.

Results: Compared to nontargeting micelles, targeting PLZ4 micelles (23.2 $\pm 8.1 \mathrm{~nm}$ in diameter) loaded with the imaging agent DiD and the chemotherapeutic drug paclitaxel or daunorubicin were more efficient in targeted drug delivery and more effective in cell killing in vitro. PLZ4 facilitated the uptake of micelles together with the cargo load into the target cells. We also developed an orthotopic invasive dog bladder cancer xenograft model in mice. In vivo studies with this model showed the targeting micelles were more efficient in targeted drug delivery than the free dye $(14.3 \times ; P<0.01)$ and nontargeting micelles $(1.5 \times ; P<0.05)$.

Conclusion: Targeting micelles decorated with PLZ4 can selectively target dog bladder cancer cells and potentially be developed as imaging and therapeutic agents in a clinical setting. Preclinical studies of targeting micelles can be performed in dogs with spontaneous bladder cancer before proceeding with studies using human patients.

Keywords: bladder urothelial carcinoma, nanoparticle, bladder cancer-specific peptide, targeted therapy, diagnostic imaging

\section{Introduction}

The prognosis and treatment of bladder cancer has not changed significantly over the last 30 years. Nanomedicine has recently been explored as a potential platform for developing anticancer strategies, ranging from prevention to early detection, imaging/diagnosis, and therapy. ${ }^{1,2}$ Several nanotherapeutic agents such as Abraxane ${ }^{\circledR}$ and Doxil $^{\circledR}$ have been approved, but their penetration ability is limited due to their relatively large particle size (130 $\mathrm{nm}$ and $150 \mathrm{~nm}$ in diameter, respectively). ${ }^{3}$ To overcome the shortcomings of existing nanoformulations, Lou et al developed a novel micelle drug-delivery system. ${ }^{4,5}$ Telodendrimers, which are micelle-forming polymers, are composed of linear polyethylene glycol (PEG) conjugated with dendritic oligocholic acid (Figure 1A). Telodendrimers can self-assemble in an aqueous solution to form micelles that are characterized by their small size, long shelf life, and high efficiency 


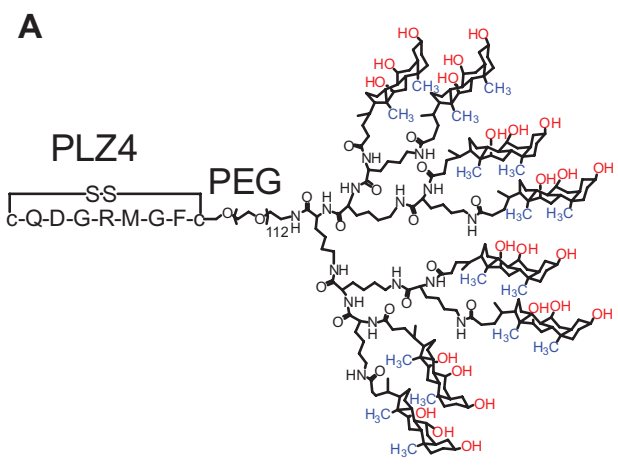

\section{B}

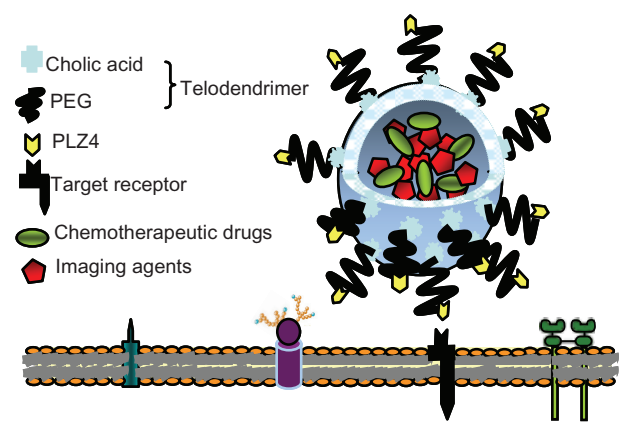

C
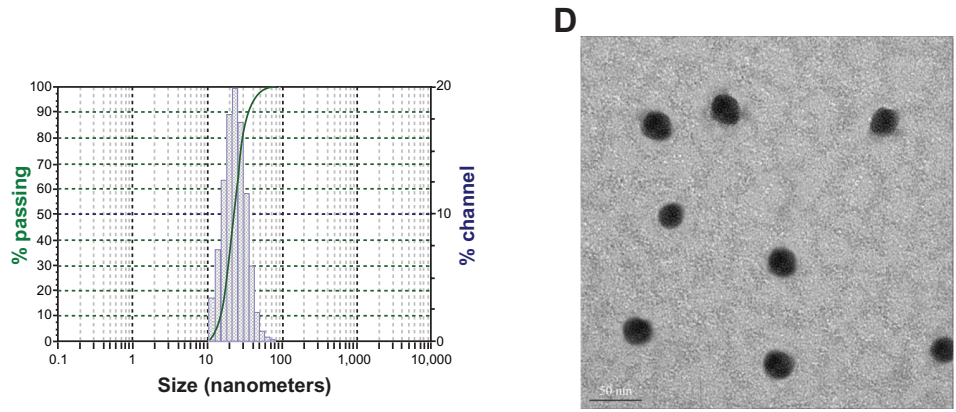

Figure I Characterization of targeting micelles. (A) The chemical structure of the telodendrimer. Dendritic oligomers of cholic acid are conjugated to the right ends of polyethylene glycol (PEG) while PLZ4 is at the left. The facial amphiphilic structure of cholic acid with the hydrophilic hydroxyl groups (red) at one side and hydrophobic methyl groups (blue) at the other side allows the formation of spheric micelles with PLZ4 displayed on the surface. (B) The spherical micelle structure with PLZ4 on the surface for targeting and the central space for loading of imaging and/or therapeutic agents. (C) Dynamic light scattering showing the size of targeting micelles (23.2 $\pm 8.1 \mathrm{~nm}$ ) with narrow distribution. (D) Transmission electron microscopy imaging.

Note: Bar length is $50 \mathrm{~nm}$.

in drug loading. PEG is biocompatible in that it has been extensively used in clinical settings, and cholic acid exists naturally in humans. Furthermore, these micelles are multifunctional. Multiple imaging and therapeutic agents can be loaded and/or conjugated on the same micelles for various purposes. Smaller micelles $(17-60 \mathrm{~nm}$ ) also have better drug delivery to tumor sites compared to larger $(>150 \mathrm{~nm})$ ones. ${ }^{4}$ Finally, cancer-targeting ligands can be conjugated to the distal end of PEG so they will be displayed on the surface of micelles to enhance drug delivery into the cancer cells.

We recently reported the development of PLZ4, a bladder cancer-targeting ligand from high throughput screening of combinatorial cyclic peptide libraries. ${ }^{6}$ It is the first such ligand that has high affinity and selectivity toward both human and dog urothelial cancer cell lines as well as cancer cells from clinical specimens. ${ }^{6,7}$ It does not bind to normal human or dog urothelial cells, blood cells, fibroblasts, human vascular endothelial cells, or other cell types. In vivo targeting/homing studies have shown that the binding of PLZ4 to bladder cancer xenografts is specific. In this study, we determined if PLZ4 enhances the tumor targeting of micelles to dog bladder cancer cells. If so, preclinical studies (including efficacy, pharmacology, and toxicology studies) can be performed in companion dog patients with spontaneous bladder cancer instead of the traditional but less pathophysiologically-relevant mouse xenograft models.

\section{Materials and methods Synthesis and characterization of micelles} Both PLZ4 (amino acid sequence: cQDGRMGFc; uppercase letters represent L-amino acids and lowercase letters represent unnatural D-cysteines used to cyclize and stabilize PLZ4) and telodendrimers were synthesized and characterized, including the chemical structure, purity, and cholic acid conjugation, as previously described (Figure 1A). ${ }^{4-6}$ In brief, the dendritic octamer of cholic acid was conjugated onto linear PEG via solution-phase condensation reactions. To synthesize targeting micelles in which $50 \%$ of the telodendrimers were conjugated to PLZ4, an aqueous-phase 'click' chemistry catalyzed by cuprous ions was used to couple the alkyne group on PLZ4 peptides to the azide group at the end of the PEG on the telodendrimer at a molar ratio of 1:2 (PLZ4:PEG). ${ }^{8}$ After conjugation, no free PLZ4 was detected, suggesting PLZ4 has been successfully conjugated 
to the telodendrimer. The conjugation was further confirmed using proton nuclear magnetic resonance. ${ }^{1}$

To load daunorubicin (DNR), paclitaxel (PTX), or fluorescent dye $1,1^{\prime}$-dioctadecyl-3,3,3',3'-tetramethylindodica rbocyanine (DiD) dye (Invitrogen, Carlsbad, CA), into the micelle, DNR/PTX/DiD (single reagent or a combination) and the telodendrimer $(20 \mathrm{mg})$ were dissolved in chloroform $(5 \mathrm{~mL})$ in a $10-\mathrm{mL}$ flask. The chloroform was removed using a rota-evaporator under vacuum. One $\mathrm{mL}$ of USP (United States Pharmacopeia) saline was then added into the flask. The mixture was vortexed and sonicated for 30 minutes at room temperature. The final product was analyzed for drug-loading capacity using high-performance liquid chromatography, micelle size and dispersion with a dynamic light scattering particle sizer, and transmission electron microscopy imaging. It was filtered through a filter $(0.22 \mu \mathrm{m})$ for sterilization and stored at $4^{\circ} \mathrm{C}$ for further studies. The maximal loading capacity was $5 \mathrm{mg} / \mathrm{mL}$ for DNR and $5-10 \mathrm{mg} / \mathrm{mL}$ for PTX in $20 \mathrm{mg} / \mathrm{mL}$ of the telodendrimer. For the experiments presented here, the final concentration of DiD was $0.5 \mathrm{mg} / \mathrm{mL}$, and the final concentrations of DNR and PTX were $1 \mathrm{mg} / \mathrm{mL}$, with a telodendrimer concentration of $20 \mathrm{mg} / \mathrm{mL}$. To synthesize targeting micelles, PLZ4-conjugated telodendrimers were used. After self-assembly, the more hydrophilic PLZ4 was displayed on the surface of the micelles for targeting.

\section{Cell lines and culture condition}

Three established and well-characterized dog invasive bladder urothelial cancer cell lines, including K9TCC, K9TCCPu-In, and K9TCC-Pu-Axc, were used for this study. ${ }^{9}$ These cell lines were originally developed and directly provided by Dr Deborah Knapp of Purdue University in July, 2009. Cells were maintained in Dulbecco's modified Eagle's medium/ F12 (1:1) (Invitrogen) supplied with 10\% fetal bovine serum and antibiotics. Normal primary dog urothelial cells were harvested from dogs euthanized for other unrelated reasons. A very thin layer of urothelial cells was scraped and washed with phosphate-buffered solution. The cells were then directly cultured on eight-chamber slides in the complete medium for 1-2 days before the experiment.

\section{Fluorescence microscopy}

To visualize the distribution of micelles in the cells, K9TCC$\mathrm{Pu}$-In cells and normal dog urothelial cells were cultured in the eight-well chamber slides (BD Falcon, Franklin Lakes, NJ). The cells were then incubated with nontargeting micelles or targeting micelles with PLZ4 at $0.5 \mathrm{mg} / \mathrm{mL}$ telodendrimers in the complete medium. After incubation, the cells were washed to remove unbound micelles and covered with 4',6-diamidino-2-phenylindole (DAPI)-containing solution for nuclear staining (Sigma-Aldrich, St Louis, MO). The slides were then examined under an inverted fluorescence microscope. For high resolution cell imaging, K9TCC-PuAxc cells were cultured on a glass-bottom culture dish (MatTek Corp, Ashland, MA) overnight. Micelles were diluted to $0.5 \mathrm{mg} / \mathrm{mL}$ telodendrimers in the complete medium and were incubated with micelles for 1 hour. After being lightly washed three times and fixed with $10 \%$ formalin for $20 \mathrm{~min}-$ utes, the cells were stained with DAPI and examined. Highresolution three-dimensional microscopy was performed using the DeltaVision system (Applied Precision, Issaquah, WA). Tomography images were taken and then deconvolved to determine the subcellular location of micelles with red fluorescence from DiD using a known optical transfer function and DeltaVision software algorithms, according to the manufacturer's protocol.

\section{Cell viability assay}

K9TCC-Pu-In cells were seeded into 96-well plates at the final concentration of 10,000 cells per well in $100 \mu \mathrm{L}$ of complete medium. The cells were incubated with various concentrations of nontargeting or targeting micelles loaded with DNR for 2 hours before being washed three times with phosphate-buffered solution and cultured with $100 \mu \mathrm{L}$ of fresh complete medium for 72 hours at $37^{\circ} \mathrm{C}$. The 2-hour incubation was used to mimic the in vivo clearance of micelles. Based on our pilot study, dog urothelial cancer cell lines appeared to be more resistant to PTX compared to human bladder cancer cell line 5637. Therefore, this study was performed with micelles loaded with DNR instead of PTX. Cell viability was assessed using the WST-8 proliferation assay (Cayman Chemical, Ann Arbor, MI) according to the manufacturer's protocol.

\section{Generation and characterization of an orthotopic mouse model of dog bladder cancer}

All animal experiments were performed in compliance with institutional guidelines and according to protocols (No. 12988 and No. 16065) approved by the Animal Use and Care Administrative Advisory Committee of the University of California (Davis, CA). Thirteen 8-week-old male nonobese diabetic severe combined immunodeficient gamma mice were purchased from Jackson Laboratory (Indianapolis, IN) and used to generate the orthotopic model. The mice were anesthetized using an intraperitoneal injection of pentobarbital or 
by isoflurane inhalation. The lower abdomen was prepared for surgery by proper scrubbing with an ethanol wipe. A small incision was performed at the bladder region and the bladder was isolated. K9TCC-Pu-In $\left(10^{5}\right.$ cells in $10 \mu \mathrm{L}$ phosphatebuffered solution) was injected into the bladder wall with a 26 $\mathrm{G}$ needle. A white focal spot stood for a successful injection procedure. After the injection, the abdomen was closed with the appropriate number of interrupted sutures, and the mice were monitored daily for wound healing, potential complications, body weight change, and tumor growth.

For histologic examination, the mice were sacrificed at days 1,7 , and 20 after injection. Whole bladders were harvested and fixed with $10 \%$ formalin. Tissue embedding and hematoxylin and eosin staining were done at the Veterinary Teaching Animal Hospital. Regarding the cytological evaluation, the urine sediment smears and touch preparation smears were made and slides were stained with Hema3 (Fisher, Houston, TX). Imaging was performed using Metamorph microscopy automation and imaging analysis software (version 2.0; Molecular Devices, Silicon Valley, CA).

\section{In vivo and ex vivo imaging of orthotopic xenograft dog bladder cancer in mice}

The mice were injected with $100 \mu \mathrm{L}$ of free $\operatorname{DiD}(0.5 \mathrm{mg} / \mathrm{mL})$, nontargeting, or targeting micelles with PLZ4 on the surface, both loaded with DiD at $0.5 \mathrm{mg}$ per $10 \mathrm{mg}$ telodendrimer per $\mathrm{mL}$ with a total of $0.05 \mathrm{mg}$ DiD per mouse. Three mice for each group in two independent experiments were used. The mice were put under anesthesia and the bladders were isolated outside the abdomen for in vivo imaging. Whole-body imaging was performed and analyzed at $0,2,4,8$, and 24 hours using a Kodak multimodal-imaging system IS2000MM (Kodak, Rochester, NY). After the imaging, the mice were immediately euthanized. Whole bladders with tumors and other organs were harvested for ex vivo imaging. Imagines were further analyzed using IS2000MM software (Kodak).

\section{Statistics}

The experiments were repeated at least in triplicate. The mean values and standard deviations were determined for each set of experiments. For the determination of micelle delivery to tumor sites, we calculated mean fluorescence intensities of the tumor area and of the normal tissue area by means of the region-of-interest function using Kodak Image Analysis Software (Kodak). We then plotted a pseudocolored scale based on the semiquantitative information from near-infrared fluorescence images by integrating fluorescence intensities from equal areas within tumor and normal tissue regions.
Student's $t$-tests were used for statistics. A $P$ value of less than 0.05 was considered significant.

\section{Results}

\section{Characterization of drug-loaded targeting micelles decorated with PLZ4}

We synthesized two types of micelles: the nontargeting micelles that did not have PLZ4 on the surface and targeting micelles that were decorated with PLZ4 on the surface (Figure 1B). Both types of micelles were loaded with the near-infrared imaging agent DiD and the therapeutic agent PTX or DNR. The median diameter of the micelles, with or without PLZ4 on the surface, was approximately $23.2 \pm 8.1 \mathrm{~nm}$ when loaded with PTX and DiD (Figure 1C). The spherical morphology and size of the micelles were confirmed by electronic microscopy (Figure 1D). The maximal drug loading capacity was $5 \mathrm{mg} / \mathrm{mL}$ for DNR and $10 \mathrm{mg} / \mathrm{mL}$ for PTX in $20 \mathrm{mg} / \mathrm{mL}$ of telodendrimer. Considering the doses of DNR and PTX at 45-60 mg/m $/ \mathrm{m}^{2}$ and $175 \mathrm{mg} / \mathrm{m}^{2}$ in human patients, respectively, the anticipated blood level of the telodendrimer will be well below the toxic level of $1 \mathrm{mg} / \mathrm{mL}$ observed in cell culture. ${ }^{5}$

\section{Targeting micelles effectively delivered the cargo load into dog bladder cancer cells}

To evaluate delivery efficiency, K9TCC-Pu-In (Figure 2A), K9TCC (Figure 2B), and K9TCC-Pu-Axc (Figure 2C) cells were incubated with micelles for 1 hour before washing. Both nontargeting and targeting micelles were able to deliver DiD to all these three cell lines in a dose-dependent manner. However, targeting micelles coated with PLZ4 exhibited significantly higher DiD. To further validate this finding, these cells were examined under a fluorescence microscope. K9TCC-Pu-In cells were treated with both nontargeting or targeting micelles loaded with $\mathrm{DiD}(50 \mu \mathrm{g} / \mathrm{mL}$ DiD in $2 \mathrm{mg} / \mathrm{mL}$ telodendrimer). K9TCC-Pu-In took up more targeting micelles than nontargeting micelles. The uptake could be observed as early as 15 minutes after incubation (data not shown). No significant uptake of micelles by normal primary dog bladder urothelial cells was observed (Figure 3A). This data indicated the ability of dog bladder cancer cells to take up micelles even after a short period of incubation, and PLZ4 on the micelle surface further improved the targeting and cell uptake of micelles. Furthermore, the targeting micelles could specifically deliver the drug load to cancer cells, but not to normal urothelial cells. 

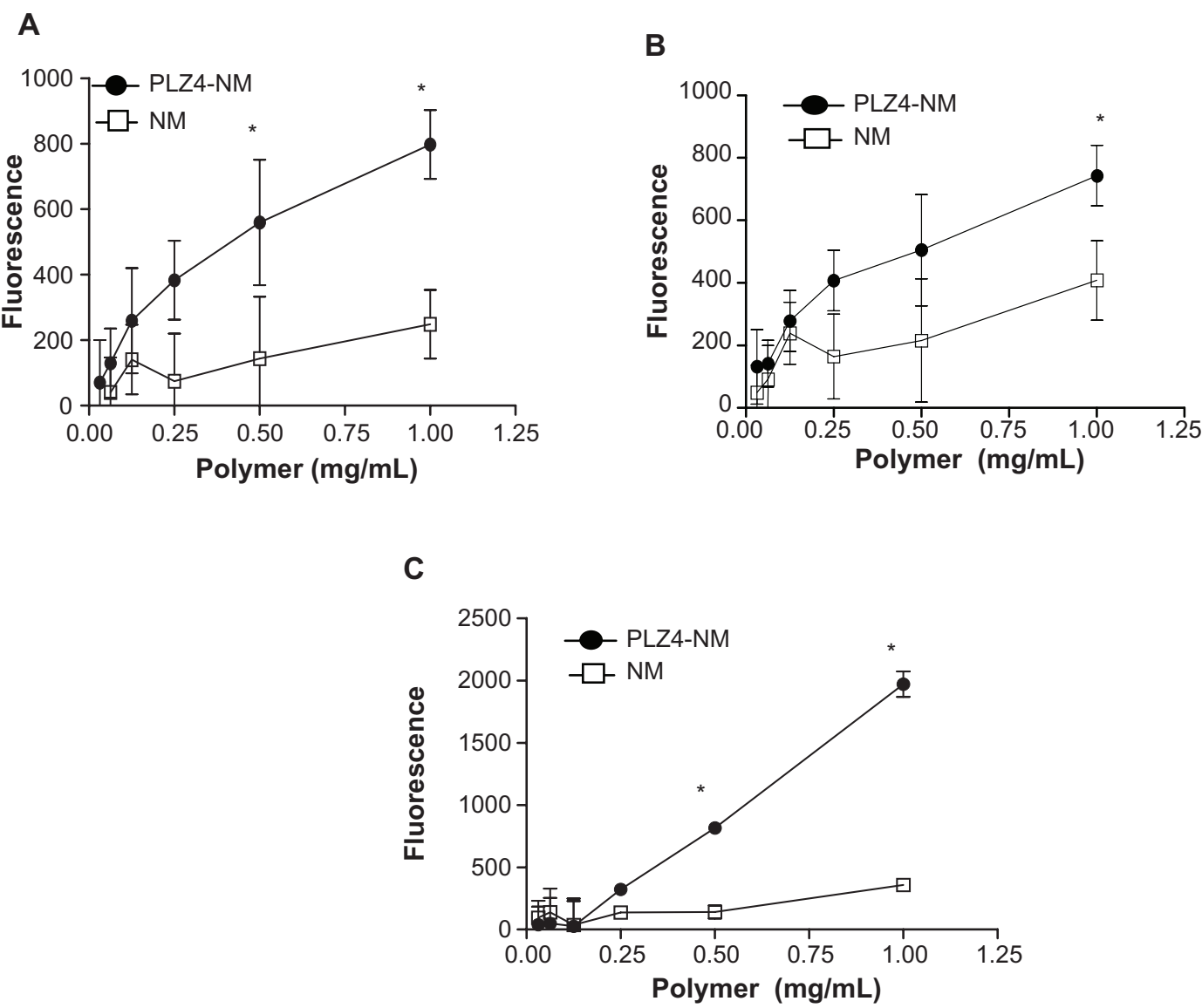

Figure 2 More efficient delivery of targeting micelles into bladder cancer cell lines. (A) K9TCC-Pu-In, (B) K9TCC, and (C) K9TCC-Pu-Axc cells were incubated with various concentrations of nontargeting (NM) or targeting (PLZ4-NM) micelles, both loaded with DiD, for I hour before washing and analysis using an enzyme-linked immunosorbent assay reader.

Note: Targeting micelles were more efficient in drug delivery than nontargeting micelles in a dose-dependent pattern (*P<0.05).

Abbreviation: DiD, I, I'-dioctadecyl-3,3,3',3'-tetramethylindodicarbocyanine.

To further determine whether PLZ4 functioned to attach micelles to the target cells or to induce the uptake of micelles, high-resolution tomography was performed using the DeltaVision system (Applied Precision) after cells were incubated with nontargeting or targeting micelles, both simultaneously loaded with DiD and PTX. After incubation for 1 hour, K9TCC-Pu-In cells were washed, fixed, and covered with DAPI-containing mounting medium for nuclear staining. As shown in Figure 3B, little uptake of DiD was observed when cells were incubated with nontargeting micelles. In contrast, when targeting micelles were incubated with cells, large amounts of red fluorescence were observed not only on cell surface and perinuclear areas, but also inside the nucleus as it overlapped with the DAPI (blue) nuclear staining (Figure 3B, upper panel). These results were consistent with several other reports showing preferential drug delivery with targeting nanoparticles. ${ }^{10,11}$ These results also support the notion that PLZ4 enhances nanoparticle attachment to the cell surface and subsequent cell uptake.

\section{PLZ4 enhanced the anticancer efficacy of drug-loaded micelles}

To determine if the drug-loaded micelle formulation further increased antitumor efficacy, K9TCC-Pu-In cells were exposed to different concentrations of DNR-loaded nontargeting or targeting micelles. Consistent with our previous findings, empty micelles did not affect cell viability at any concentrations tested. DNR killed cancer cells in a dose-dependent manner, regardless of whether in the free form, nontargeting, or targeting micelle formulation. However, the targeting micelle formulation of DNR was more effective in cell killing than the free DNR or DNR loaded in the nontargeting micelles (Figure 4).

\section{Establishment and characterization of an orthotopic dog bladder cancer xenograft model in mice}

An orthotopic xenograft model would be essential to validate our micelle drug delivery system, as the location provides a 


\section{A}

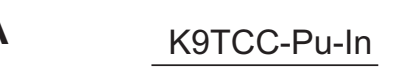

$\underline{\text { Dog primary urothelial cells }}$
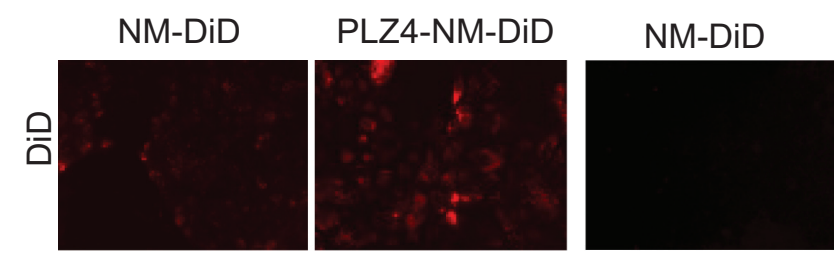

PLZ4-NM-DiD
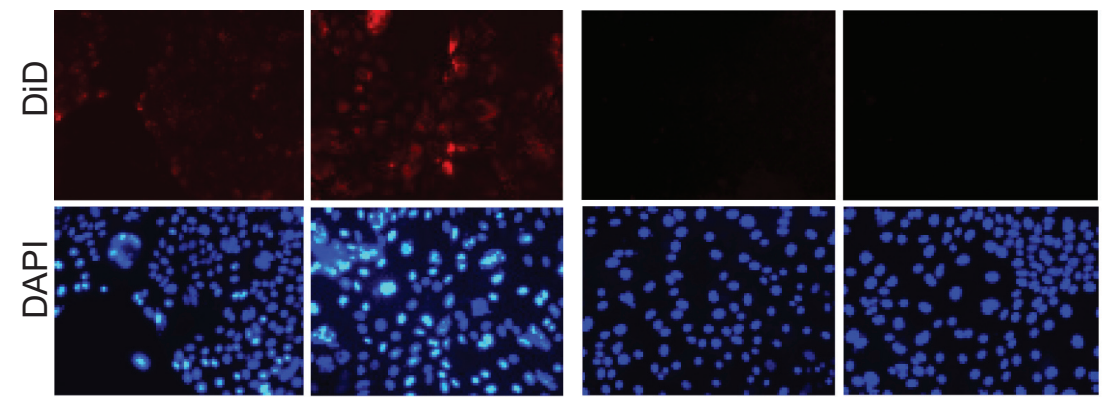

B
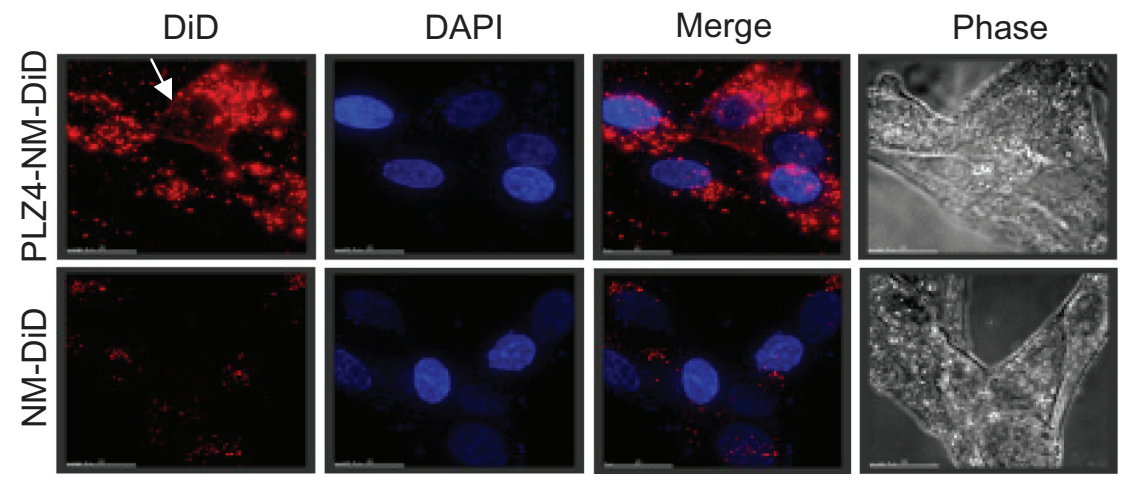

Figure 3 Cell uptake of nontargeting and targeting micelles by dog bladder cancer cells. K9TCC-Pu-In cells were incubated with nontargeting (NM-DiD) or targeting micelles (PLZ4-NM-DiD), both loaded with DiD/PTX, for I hour before washing and imaging. (A) Comparison of cell uptake between nontargeting and targeting micelles with K9TCC-Pu-In (left) and normal dog primary urothelial cells (right) showing little retention of micelles with normal urothelial cells. The experiments were repeated three times with the cell line and twice with primary urothelial cells (I00x). (B) High-resolution tomography showed the difference in the cell uptake of targeting (PLZ4-NM-DiD, upper panel) and nontargeting (NM-DiD, lower panel) micelles in K9TCC-Pu-Axc cells. 4',6-diamidino-2-phenylindole (blue, nuclear staining) and white light phase images were presented to show nucleus and cell morphology, respectively.

Notes: The white arrow points to the attachment of targeting micelles to the cell membrane. Bar $=15 \mu \mathrm{m}$.

Abbreviations: DiD, I, I'-dioctadecyl-3,3,3',3'-tetramethylindodicarbocyanine; PTX, paclitaxel.

blood vessel system and supporting tissues that mirror the native cancer microenvironment. Here, we developed the orthotopic dog invasive bladder cancer xenograft model in mice through direct injection of K9TCC-Pu-In cells into the bladder wall. The mice tolerated the procedure well, with no significant complications. The tumor mass in the bladder became palpable approximately $2-3$ weeks after injection. To further evaluate the growth of the orthotopic xenografts in the mouse bladder in detail, whole bladders were harvested at days 1,7 , and 20 after injection. Overall, the tumor uptake rate was $100 \%(13 / 13)$ based on pathological examination and tumor size ranged around $0.3-0.6 \mathrm{~cm}$ in diameter on day 20. Based on the histopathologic examination, tumor cells were mainly accumulated and limited in the lamina propria of the bladder on day 1 (Figure 5A and D). The cells were loosely clumped without organization or architecture. Rare cells were undergoing apoptosis (Figure 5D). On day 7 , the mass continuously expanded in the lamina propria and invaded the superficial layer of the muscularis propria. Several nests of epithelial cancer cells with trabecular patterns supported by abundant fibrovascular connective tissues were seen (Figure 5B). The urothelial layer remained intact, and inflammatory cells infiltrated the mass, especially in the peripheral areas (Figure 5E). On day 20 (Figure 5C and F), the mass expanded in the lamina propria and extended into the bladder cavity, resulting in extreme limitation of urine storage ability. Tumor cells also further invaded through the full layer of the muscularis propria and into the surrounding fat tissues. The mitotic index was high. Rare areas of urothelium cells were necrotic and focal hemorrhage was noted.

Once the tumor cells penetrated through the urothelial layer, hematuria, and an increased number of nucleated cells in the urine were noted after a urine sediment smear evaluation. Figure $5 \mathrm{G}$ shows a small cluster of epithelial cells in the urine in a bloody background on day 20. Those atypical cells exhibited moderate anisocytosis and anisokaryosis, with round to irregular nuclei, stippled chromatin, multiple 


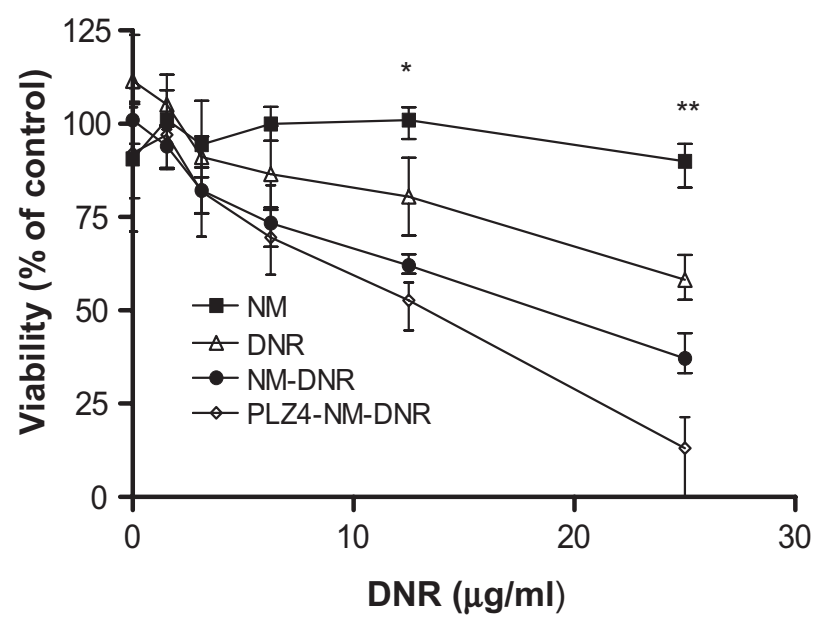

Figure 4 Cell cytotoxicity assay of drug-loaded micelles on dog bladder cancer. K9TCC-Pu-In cells were seeded and cultured overnight before being treated with various concentrations of nontargeting or targeting micelles loaded with daunorubicin (DNR) for two hours at $37^{\circ} \mathrm{C}$. Cells treated with empty micelles and free DNR under the same conditions served as controls. Cells were then washed and cultured with complete culture medium for 72 hours and the cell viability was evaluated using the WST- 8 assay according to the manufacturer's protocol. The absorbance was detected using an enzyme-linked immunosorbent assay reader. This experiment was performed in triplicate and repeated three times.

Notes: $* P=0.02$ for free DNR versus PLZ4 micelles and 0.04 for free DNR versus nontargeting micelles. ${ }^{* *} P=0.00$ I for free DNR versus PLZ4 micelles; 0.01 for free DNR versus nontargeting micelles; and 0.04 for nontargeting versus targeting micelles.

nucleoli, and dark basophilic cytoplasm. Rare binucleated and multinucleated cells were seen. These cells resembled cells found on the bladder mass touch preparation slides and were diagnosed as cancer cells (Figure 5I). Rare cells contained magenta amorphous materials in the cytoplasm and were interpreted as a secretory product (a common feature of urothelial cancer cytology in dogs).

\section{In vivo micelle delivery to orthotopic dog bladder cancer xenografts in mice}

After successful establishment of the orthotopic xenograft model, we determined if nontargeting and targeting micelles, both loaded with DiD and PTX, could have accumulated within the cancer xenografts. Both micelles were able to efficiently accumulate at the tumor site compared to the free DiD control group in vivo and ex vivo, suggesting that the so-called enhanced permeability and retention (EPR) mechanism played an important role in the delivery of micelles (Figure 6A-C). The targeting micelle group showed notably higher fluorescence signals at tumor sites compared to the free dye $(14.3 \times ; P<0.01)$ or nontargeting group $(1.5 \times ; P<0.05$; Figure $6 \mathrm{~A}-\mathrm{C})$. After normalizing tumor fluorescence with fluorescence in the liver, lungs, and muscles (Figure 6D), the results were generally consistent with the in vivo imaging findings. In mice that did not undergo orthotopic bladder cancer xenografting, no signal in the bladder was seen (data not shown), suggesting that only a limited number of DiD-loaded nontargeting or targeting micelles accumulated within normal bladder mucosa.

To further evaluate the distribution of micelles, fresh frozen slides were prepared and stained for CD31, a marker for vascular endothelial cells (Supplementary material 1). No signals were observed in the group treated with DiD. In contrast, both groups treated with nontargeting or targeting micelles exhibited patches of fluorescence signals with variable sizes. Xenografts from the mice treated with targeting micelles appeared to have higher signals than the nontargeting group. This finding was consistent with the in vivo and ex vivo imaging results stated above. In addition, blood vessels (CD31 positive) were scattered throughout the tumors, mainly in the fibrovascular-supporting tissues in all three groups, indicating that the difference was not from the variance in blood vessel distribution. The distribution of DiD was not homogenous inside the tumor, as evidenced by the variably sized signal patches, suggesting that delivery of DiD to tumor sites was not secondary to blood circulation and simple diffusion.

\section{Discussion}

This is the first report on the development of bladder cancertargeting nanoparticles that can potentially improve the imaging detection and treatment of bladder cancer. This project combined a bladder cancer-targeting ligand, PLZ4, with a novel micelle drug delivery system. When decorated with PLZ4 on the surface, targeting micelles could not only adhere to the dog bladder cancer cell surface, but were also taken up into the target cancer cells. In vivo studies showed that the targeting micelles were more efficient in delivering the therapeutic and imaging agents into the orthotopic bladder cancer xenografts. Given the great similarities between human and dog bladder cancers, this will allow all of the preclinical studies (to human patients) to be performed in dogs with spontaneous bladder cancer before clinical trials in human patients.

The unique advantage of the micelle system is that multiple imaging and therapeutic agents can be conjugated to telodendrimers or loaded in micelles (Figure 1A). When decorated with PLZ4, these imaging agents can be specifically delivered to the cancer sites. Targeted delivery of imaging agents has already been used in clinical settings. For example, an octreoscan combines the diethylene triamene penta-acetate conjugate of octreotide with the imaging agent radioisotope Indium-111, and has been used for imaging detection of 
Day 1
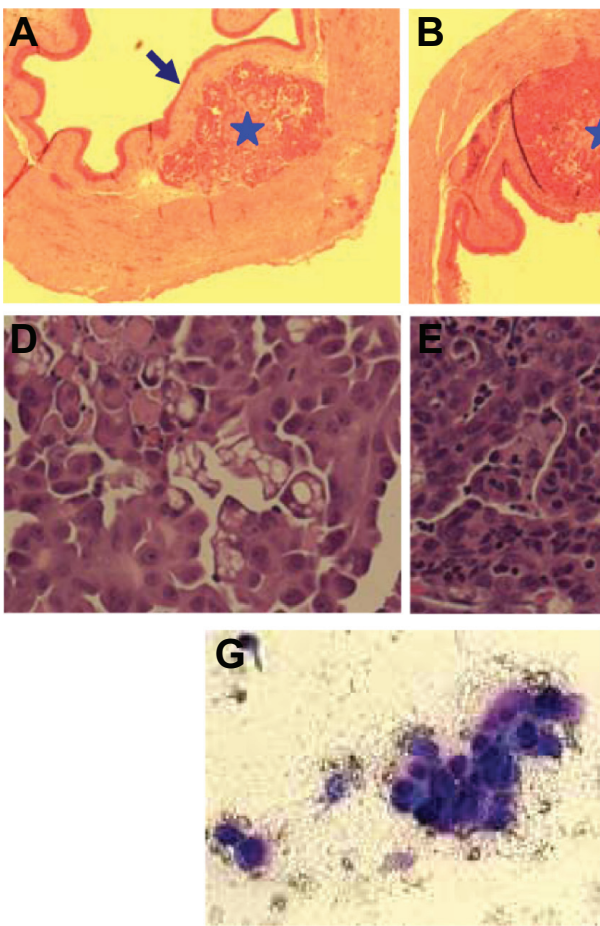

Day 7
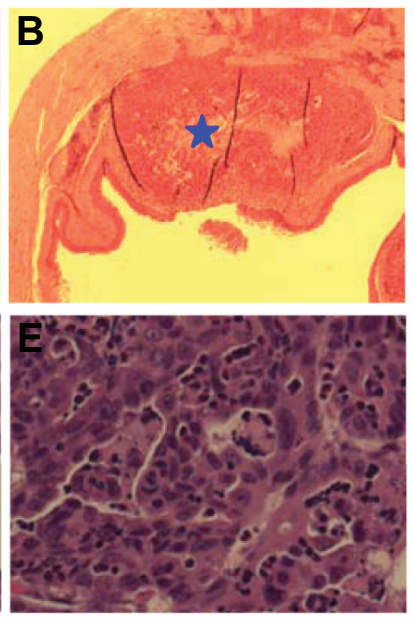

Day 20
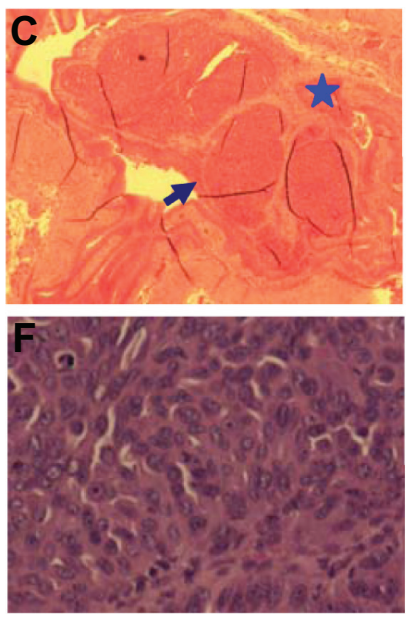

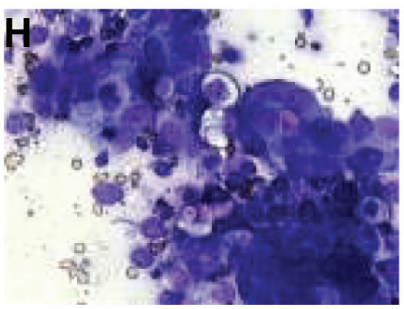

Figure 5 Establishment of dog orthotopic bladder cancer xenograft model in mice. Whole bladders were harvested on days I (A and D), 7 (B and E), and 20 (C and $\mathbf{F}$ ) after injection of cancer cells for hematoxylin and eosin staining and examination. Blue arrows show an intact normal urothelial layer lining the bladder cavity on day I and replacement of the normal urothelial layer and bladder cavity by xenograft on day 20. Blue stars show the injection (day I) and expansion of cancer cells (day 7 ) at the lamina propria and invasion into the muscle layer on day 20. (A, B and C: $4 \times ; \mathbf{D}, \mathbf{E}$, and F: 40×). (G) urine sediment cytology evaluation on day 20 (40×). (H) The touch preparation smear from the solid tumor in the bladder at day $20(40 \times)$.

neuroendocrine tumors bearing somatostatin receptors. ${ }^{12} \mathrm{We}$ have previously shown that PLZ4 directly conjugated to a near-infrared fluorescent dye Cy5.5 concentrated in the tumor xenograft sites in mouse models developed from human clinical bladder cancer specimens and from dog bladder cancer cell lines. ${ }^{6,7}$ No significant accumulation in other organs, including the native bladder, was observed except that both PLZ4-Cy5.5 and the control dye Cy5.5 also concentrated in the kidneys, possibly secondary to nonspecific renal trapping. Here, we showed that targeting PLZ4 micelles loaded with the imaging agent DiD also concentrated in the bladder in the tumor xenografts (Figure 6). However, we did see some uptake of micelles in the liver and lungs. However, the fluorescence in the tumor xenografts was much stronger. It has also been shown that the nonspecific uptake of micelles by normal organs could be significantly reduced using micelles with a slight negative charge on the surface. ${ }^{13}$ Together, these data suggest that targeting micelles can potentially be developed as a more efficient, noninvasive, real-time imaging diagnostic tool.

The targeting micelle decorated with PLZ4 can also be used for therapeutic purposes. Both nontargeting and targeting micelles concentrated within tumor xenografts, suggesting the enhanced EPR of nanoparticles. ${ }^{14}$ However, conjugation of PLZ4 on the surface of micelles not only contributed to the attachment of micelles to target cancer cell surfaces, but also induced the uptake of micelles by cancer cells (Figure 3 ). Furthermore, PLZ4 enhanced targeted delivery of micelles to the orthotopic bladder cancer xenografts (Figure 6).

This micelle drug delivery system can potentially improve the treatment outcomes of bladder cancer through four possible mechanisms. First, micelles can enhance drug delivery to the tumor sites through the EPR effect. ${ }^{14}$ EPR is achieved at the "leaky" neovasculature secondary to the disorganized and poorly aligned vascular endothelial cells at the tumor sites. ${ }^{15}$ We have shown that the EPR effect was size-dependent. More EPR effects were observed with small micelles (20-60 nm) compared to nanoparticles of approximately $150 \mathrm{~nm} .{ }^{4}$ Compared to the FDA-approved Abraxane ${ }^{\circledR}$ and Doxil ${ }^{\circledR}(130 \mathrm{~nm}$ and $150 \mathrm{~nm}$ in diameter, respectively), our drug-loaded micelles are typically small $(23.2 \pm 8.1 \mathrm{~nm}$, in this case. See Figure 1).

Second, tumor-targeting ligands can further enhance the antitumor activity of nanoparticles. ${ }^{16}$ To enhance the 
A

DiD NM-DiD PLZ4-NM-DiD
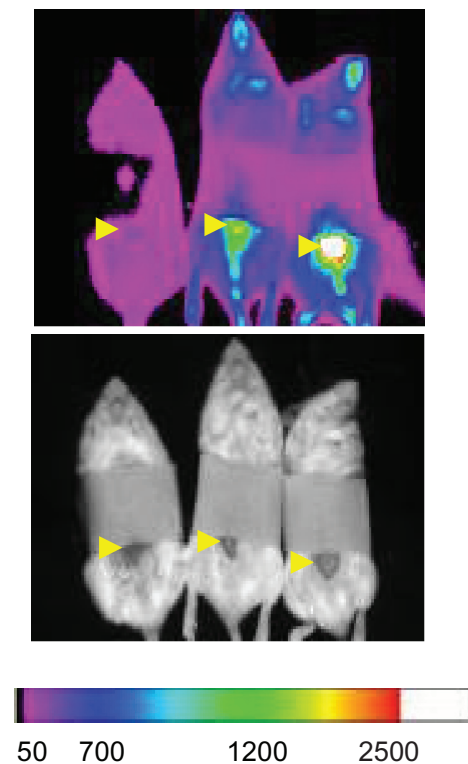

B

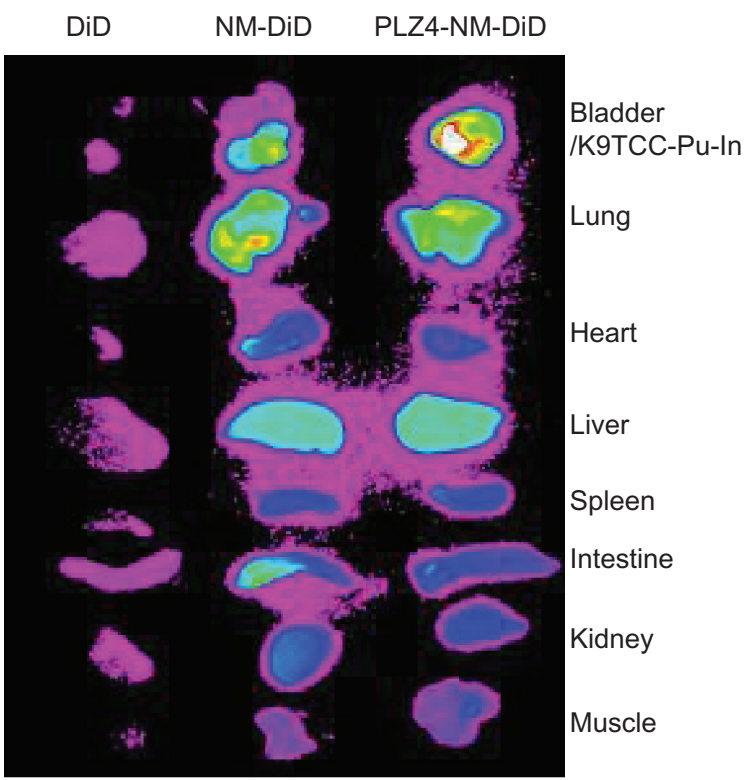

C

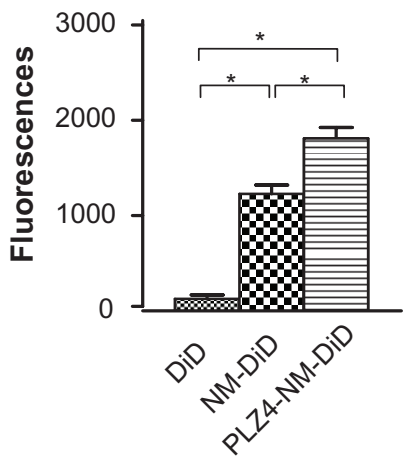

D
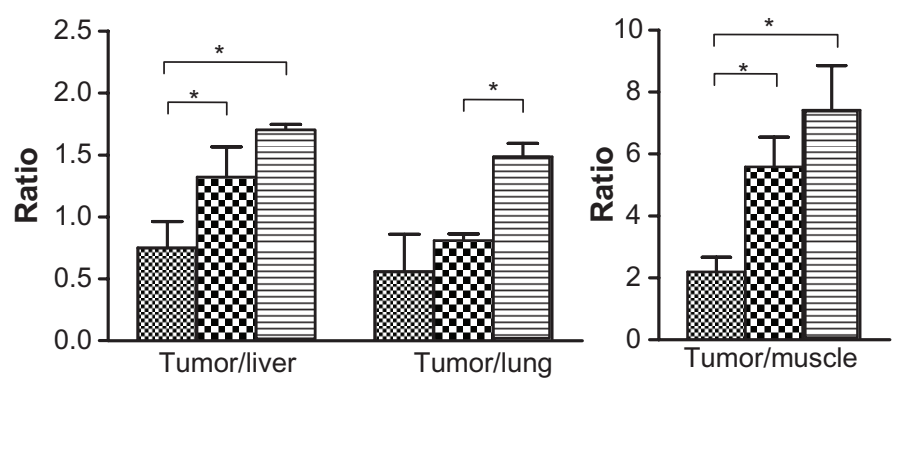

\% DiD

$\infty$ NM-DiD

뗑 PLZ4-NM-DiD

Figure 6 In vivo and ex vivo imaging on an orthotopic dog invasive bladder cancer model in mice. (A) In vivo imaging of mice 24 hours after receiving free DiD dye or nontargeting (NM-DiD) or targeting (PLZ4-NM-DiD) micelles loaded with PTX/DiD. Mice were covered to protect bladder xenografts and to prevent evaporation of vital organs. (B) Ex vivo imaging of the tumor/bladder and other major organs 24 hours after injection. The color bar represents the relative fluorescence strength. (C) The average fluorescence strength of whole tumor/bladder for free DiD, NM-DiD, and PLZ4-NM-DiD was calculated from three different mice (*P $<0.05)$. (D) To eliminate other variations, the fluorescence signals of xenografts were normalized with the signals of the liver, lungs, and muscles of the same mice $(* P<0.05)$.

Abbreviations: DiD, I, I'-dioctadecyl-3,3,3',3'-tetramethylindodicar bocyanine; PTX, paclitaxel.

antitumor activity of the nanoformulation, various targeting ligands have been studied, such as arginine-glycine-aspartic acid-containing peptides, ${ }^{17}$ transferrin, ${ }^{18,19}$ antibody or singlechain variable fragments, ${ }^{20}$ and several others. These cancertargeting ligands could potentially enhance the antitumor efficacy of the nanoparticles by actively seeking and delivering nanoparticles to the cancer sites, ${ }^{21,22}$ or by introducing the nanoparticles into cancer cells. ${ }^{23-25}$ Here, we showed that PLZ4-decorated micelles possess both features of targeting nanoparticles (Figures 3 and 6).

Third, a synergistic effect can be achieved by loading multiple anticancer agents in the same micelles.
Furthermore, a radioisotope can be conjugated to achieve concurrent chemoradiation, and imaging probes can be loaded for the in vivo real-time monitoring of drug delivery. To date, more than a dozen drugs have been successfully loaded into micelles. In this project, we loaded the micelles with both therapeutic PTX and optical imaging DiD. In addition, other biologic compounds, such as DNA and RNA, can potentially be loaded. We found that small noncoding regulatory microRNA might be involved in the chemoresistance of bladder cancer. ${ }^{26}$ Therefore, the simultaneous delivery of therapeutic agents and chemotherapy sensitizers into cancer cells may be able to improve the treatment outcomes of bladder cancer. 
Fourth, the formulation of chemotherapeutic drugs in micelles can decrease toxicity. This is especially true with PTX as it is not very water soluble and needs a special solvent composed of Cremophor ${ }^{\circledR}$ EL (polyoxyethylated castor oil) and dehydrated alcohol. This solvent can cause severe allergic reactions and requires corticosteroid and antihistamine premedications. We have previously found that PTX in the micelle formulation at three times the therapeutic dose had a similar toxicity to the parental PTX in mouse models, but with vastly superior therapeutic effects. ${ }^{4}$

The dog orthotopic xenograft model developed for this study closely resembles human invasive bladder cancer stage T2 (invading the muscularis propria) and stage T3 (extending to tissue outside the bladder) and thus serves as a great model for both diagnostic imaging and targeted therapy for this disease. Several other bladder cancer models have been developed. However, those models resemble superficial bladder cancer. ${ }^{27,28}$

Our findings suggest that PLZ4 micelles can potentially be developed for diagnostic and therapeutic applications in dog bladder urothelial carcinoma. Dog bladder cancer is a clinical challenge in terms of early diagnosis and treatment. It is the most common bladder cancer in dogs and accounts for over $85 \%$ of urinary tract neoplasms. ${ }^{29}$ Current treatment has not been very successful due to the nature of cancer invasiveness, high metastasis rates $(37 \%-50 \%$ at diagnosis), and poor responses to chemotherapy. ${ }^{30,31}$ Even with a multimodal combination of chemotherapy, surgery, and radiotherapy, the prognosis is poor. Therefore, a better and more specific targeting carrier system may be helpful for disease management.

In summary, we have presented a novel multifunctional targeting micelle delivery system that can potentially be developed for imaging and therapeutic applications in dog bladder cancer. Considering the great similarity between dog and human bladder cancer, and that PLZ4 also binds to human bladder cancer cells, the PLZ4 micelle system has the potential for human applications. Based on this study, a Phase I clinical trial is currently being developed to determine the efficacy and toxicity of paclitaxel-loaded targeting micelles in dog patients with spontaneous bladder cancer.

\section{Acknowledgments}

We would like to thank Dr Deborah Knapp at Purdue University for providing the dog bladder cancer cell lines for this study, and Dr Lane and Dr Yao for their kindness in sharing the microscopic digital imaging system for the in vitro study and the cryosection that was performed at the UC Davis Children's Hospital. Special thanks go to Dr Kai Xiao and Dr Yen-Ju Wang for their comments about and technical assistance with the experiments. This study was supported by the VA Career Development Award 2 (PI: Pan) NIH/NCI (R01CA115483 and R01CA140449) and NIH/NIBIB (R01EB012569) (PI: Lam).

\section{Disclosure}

The authors report no conflicts of interest in this work.

\section{References}

1. He X, Gao J, Gambhir SS, Cheng Z. Near-infrared fluorescent nanoprobes for cancer molecular imaging: status and challenges. Trends Mol Med. 2010;16:574-583.

2. Bharali DJ, Mousa SA. Emerging nanomedicines for early cancer detection and improved treatment: current perspective and future promise. Pharmacol Ther. 2010;128:324-335.

3. Senior JH. Fate and behavior of liposomes in vivo: a review of controlling factors. Crit Rev Ther Drug Carrier Syst. 1987;3:123-193.

4. Luo J, Xiao K, Li Y, et al. Well-defined, size-tunable, multifunctional micelles for efficient paclitaxel delivery for cancer treatment. Bioconjug Chem. 2010;21:1216-1224.

5. Xiao K, Luo J, Fowler WL, et al. A self-assembling nanoparticle for paclitaxel delivery in ovarian cancer. Biomaterials. 2009;30:6006-6016.

6. Zhang H, Aina OH, Lam KS, et al. Identification of a bladder cancerspecific ligand using a combinatorial chemistry approach. Urol Oncol. September 29, 2012. [Epub ahead of print.]

7. Lin TY, Zhang H, Wang S, et al. Targeting canine bladder transitional cell carcinoma with a human bladder cancer-specific ligand. Mol Cancer. 2011;10:9.

8. Tornoe CW, Christensen C, Meldal M. Peptidotriazoles on solid phase: [1,2,3]-triazoles by regiospecific copper(i)-catalyzed 1,3-dipolar cycloadditions of terminal alkynes to azides. J Org Chem. 2002;67:3057-3064.

9. Dhawan D, Ramos-Vara JA, Stewart JC, Zheng R, Knapp DW. Canine invasive transitional cell carcinoma cell lines: in vitro tools to complement a relevant animal model of invasive urinary bladder cancer. Urol Oncol. 2009;27:284-292.

10. Schiller JH, Harrington D, Belani CP, et al. Comparison of four chemotherapy regimens for advanced non-small-cell lung cancer. N Engl J Med. 2002;346:92-98.

11. Leamon CP, Reddy JA. Folate-targeted chemotherapy. Adv Drug Deliv Rev. 2004;56:1127-1141.

12. Kwekkeboom D, Krenning EP, de Jong M. Peptide receptor imaging and therapy. J Nucl Med. 2000;41:1704-1713.

13. Xiao K, Li Y, Luo J, et al. The effect of surface charge on in vivo biodistribution of PEG-oligocholic acid based micellar nanoparticles. Biomaterials. 2011;32:3435-3446.

14. Matsumura Y, Maeda H. A new concept for macromolecular therapeutics in cancer chemotherapy: mechanism of tumoritropic accumulation of proteins and the antitumor agent smancs. Cancer Res. 1986;46:6387-6392.

15. Modi S, Prakash Jain J, Domb AJ, Kumar N. Exploiting EPR in polymer drug conjugate delivery for tumor targeting. Curr Pharm Des. 2006;12:4785-4796.

16. Kim K, Kim JH, Park H, et al. Tumor-homing multifunctional nanoparticles for cancer theragnosis: Simultaneous diagnosis, drug delivery, and therapeutic monitoring. J Control Release. 2010;146:219-227.

17. Garanger E, Boturyn D, Dumy P. Tumor targeting with RGD peptide ligands-design of new molecular conjugates for imaging and therapy of cancers. Anticancer Agents Med Chem. 2007;7:552-558. 
18. Xu L, Pirollo KF, Tang WH, Rait A, Chang EH. Transferrin-liposomemediated systemic p53 gene therapy in combination with radiation results in regression of human head and neck cancer xenografts. Hum Gene Ther. 1999;10:2941-2952.

19. Daniels TR, Delgado T, Rodriguez JA, Helguera G, Penichet ML. The transferrin receptor part I: Biology and targeting with cytotoxic antibodies for the treatment of cancer. Clin Immunol. 2006;121:144-158.

20. Xu L, Tang WH, Huang CC, et al. Systemic p53 gene therapy of cancer with immunolipoplexes targeted by anti-transferrin receptor scFv. Mol Med. 2001;7:723-734.

21. Wu AM, Yazaki PJ, Tsai S, et al. High-resolution microPET imaging of carcinoembryonic antigen-positive xenografts by using a copper64-labeled engineered antibody fragment. Proc Natl Acad Sci U S A. 2000;97:8495-8500.

22. Yang L, Peng XH, Wang YA, et al. Receptor-targeted nanoparticles for in vivo imaging of breast cancer. Clin Cancer Res. 2009;15: 4722-4732.

23. Hussain S, Pluckthun A, Allen TM, Zangemeister-Wittke U. Antitumor activity of an epithelial cell adhesion molecule targeted nanovesicular drug delivery system. Mol Cancer Ther. 2007;6:3019-3027.

24. Kirpotin DB, Drummond DC, Shao Y, et al. Antibody targeting of longcirculating lipidic nanoparticles does not increase tumor localization but does increase internalization in animal models. Cancer Res 2006;66:6732-6740.
25. Pun SH, Tack F, Bellocq NC, et al. Targeted delivery of RNA-cleaving DNA enzyme (DNAzyme) to tumor tissue by transferrin-modified, cyclodextrin-based particles. Cancer Biol Ther. 2004;3:641-650.

26. Vinall RL, Zripoll A, Wang S, Pan CX, Devere White RW. MiR-34a chemo-sensitizes bladder cancer cells to cisplatin treatment regardless of P53-Rb pathway status. Int J Cancer. June 23, 2011. [Epub ahead of print.]

27. Tanaka M, Gee JR, De La Cerda J, et al. Noninvasive detection of bladder cancer in an orthotopic murine model with green fluorescence protein cytology. J Urol. 2003;170:975-978.

28. Watanabe T, Shinohara N, Sazawa A, et al. An improved intravesical model using human bladder cancer cell lines to optimize gene and other therapies. Cancer Gene Ther. 2000;7:1575-1580.

29. Norris AM, Laing EJ, Valli VE, et al. Canine bladder and urethral tumors: a retrospective study of 115 cases (1980-1985). J Vet Intern Med. 1992;6:145-153.

30. Mutsaers AJ, Widmer WR, Knapp DW. Canine transitional cell carcinoma. J Vet Intern Med. 2003;17:136-144.

31. Deborah WK, Nita WG, Dennis BD, Patty LB, Tsang LL, Lawrence TG. Naturally-occurring canine transitional cell carcinoma of the urinary bladder A relevant model of human invasive bladder cancer. Urol Oncol. 2000;5:47-59. 


\section{Supplementary figure}

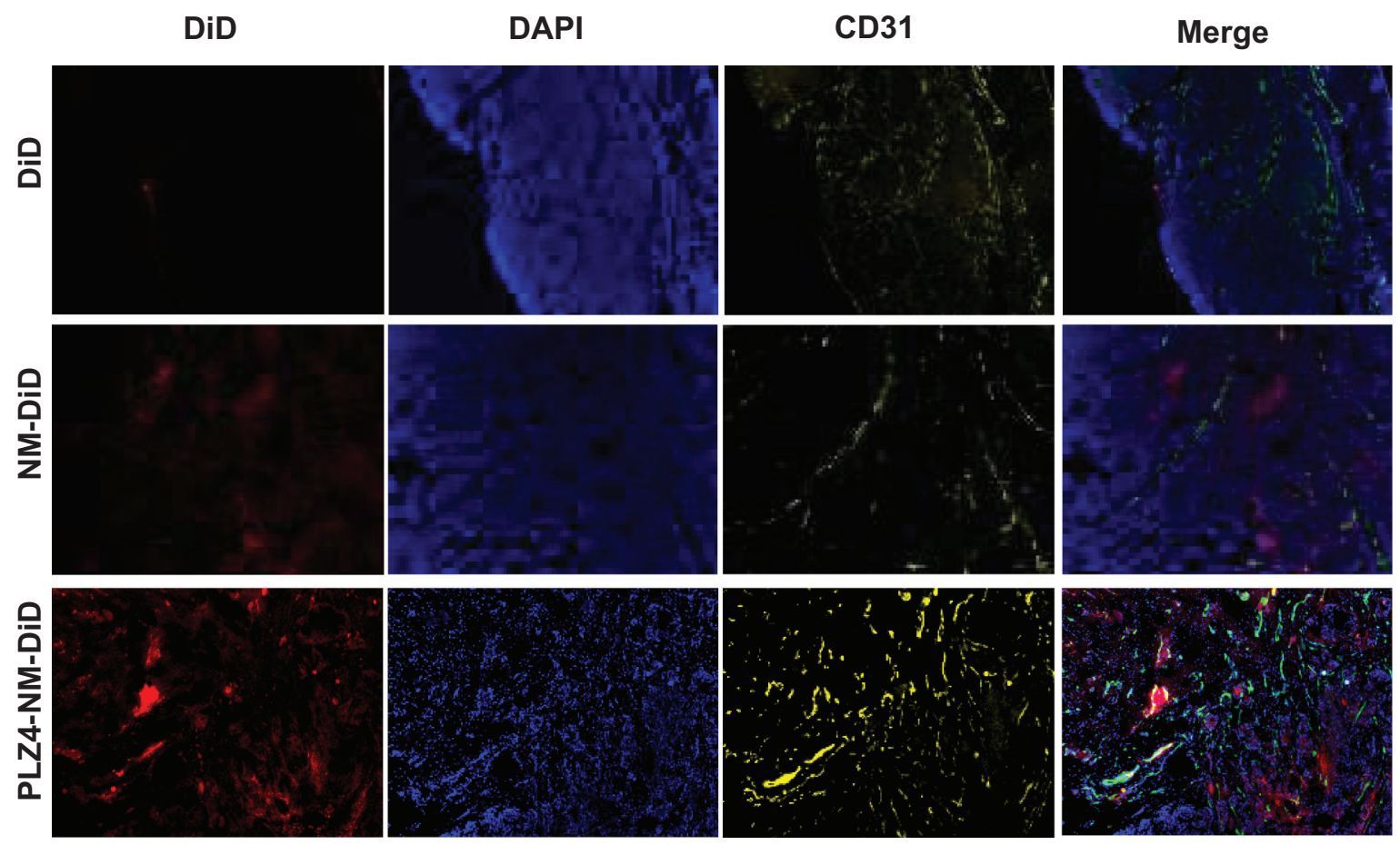

Figure SI Intratumoral distribution of micelles. After ex vivo imaging of tumor xenografts, cryosections ( $10 \mu \mathrm{m}$ thick) were obtained and the samples were fixed with $10 \%$ formalin for 10 minutes at room temperature. After 30 minutes of blocking in I\% BSA/PBS, samples were incubated with I:I00 antiCD3। (blood vessel endothelium cell marker) antibody (Millipore, Billerica, MA) for I hour at room temperature. Goat antimouse IgG conjugated Cy3 was used as a secondary antibody for another I hour at room temperature. Before observing using the microscope, slides were mounted using DAPI-containing antifading mounting medium. The imaging was acquired using Metamorph Microscopy Automation and Imaging analysis software (Molecular Devices). The distribution of DiD/micelles is shown as red, while the vascular endothelial marker $\mathrm{CD} 3 \mathrm{I}$ is shown as yellow and the nucleus/DAPI as blue in the xenografts from mice treated with free DiD (upper panel), nontargeting micelles (NM-DiD, middle panel), and PLZ4-targeting micelles (PLZ4-NM-DiD, lower panel). The very right panel shows the merged imaging.

Abbreviations: BSA/PBS, bovine serum albumin/phosphate-buffered solution; DAPI, 4',6-diamidino-2-phenylindole; DiD, I,I'-dioctadecyl-3,3,3',3'-tetramethylindodicar bocyanine.

\section{Publish your work in this journal}

The International Journal of Nanomedicine is an international, peerreviewed journal focusing on the application of nanotechnology in diagnostics, therapeutics, and drug delivery systems throughout the biomedical field. This journal is indexed on PubMed Central, MedLine, CAS, SciSearch ${ }^{\circledR}$, Current Contents $₫ /$ Clinical Medicine,
Journal Citation Reports/Science Edition, EMBase, Scopus and the Elsevier Bibliographic databases. The manuscript management system is completely online and includes a very quick and fair peer-review system, which is all easy to use. Visit http://www.dovepress.com/ testimonials.php to read real quotes from published authors. 